\title{
1 An ancient antimicrobial protein co-opted by a fungal plant pathogen for in
}

\section{2 planta mycobiome manipulation}

4 Nick C. Snelders ${ }^{1,2,3}$, Gabriella C. Petti ${ }^{2}$, Grardy C. M. van den Berg ${ }^{1}$, Michael F. Seidl ${ }^{3}$, Bart P.H.J.

$5 \quad$ Thomma $^{1,2 *}$

6

$7 \quad{ }^{1}$ Laboratory of Phytopathology, Wageningen University \& Research, Wageningen, The Netherlands

$8{ }^{2}$ Cluster of Excellence on Plant Sciences (CEPLAS), Institute for Plant Sciences, University of Cologne,

9 Cologne, Germany

$10{ }^{3}$ Theoretical Biology \& Bioinformatics Group, Department of Biology, Utrecht University, Utrecht,

11 The Netherlands

12

$13{ }^{*}$ Corresponding author: Institute for Plant Sciences, Cluster of Excellence on Plant Sciences (CEPLAS),

14 Universität zu Köln, Zülpicher Straße 47b, D-50674 Cologne, Germany. Tel: +49-221-47089261, e-

15 mail: bthomma@uni-koeln.de 


\section{ABSTRACT}

17 Microbes typically secrete a plethora of molecules to promote niche colonization. Soil-dwelling

18 microbes are well-known producers of antimicrobials that are exploited to outcompete microbial co-

19 inhabitants. Also plant pathogenic microbes secrete a diversity of molecules into their environment

20 for niche establishment. Upon plant colonization, microbial pathogens secrete so-called effector

21 proteins that promote disease development. While such effectors are typically considered to

22 exclusively act through direct host manipulation, we recently reported that the soil-borne fungal

23 xylem-colonizing vascular wilt pathogen Verticillium dahliae exploits effector proteins with

24 antibacterial properties to promote host colonization through the manipulation of beneficial host

25 microbiota. Since fungal evolution preceded land plant evolution, we now speculate that a subset of

26 the pathogen effectors involved in host microbiota manipulation evolved from ancient antimicrobial

27 proteins of terrestrial fungal ancestors that served in microbial competition prior to the evolution of

28 plant pathogenicity. Here, we show that $V$. dahliae has co-opted an ancient antimicrobial protein as

29 effector, named VdAMP3, for mycobiome manipulation in planta. We show that VdAMP3 is

30 specifically expressed to ward off fungal niche competitors during resting structure formation in

31 senescing mesophyll tissues. Our findings indicate that effector-mediated microbiome manipulation

32 by plant pathogenic microbes extends beyond bacteria and also concerns eukaryotic members of

33 the plant microbiome. Finally, we demonstrate that fungal pathogens can exploit plant microbiome-

34 manipulating effectors in a life-stage specific manner, and that a subset of these effectors has

35 evolved from ancient antimicrobial proteins of fungal ancestors that likely originally functioned in 


\section{SIGNIFICANCE STATEMENT}

38 Microbes secrete a diversity of molecules into their environment to mediate niche colonization.

39 During host ingress, plant pathogenic microbes secrete effector proteins that facilitate disease

40 development, many of which deregulate host immune responses. We recently demonstrated that

41 plant pathogens additionally exploit effectors with antibacterial activities to manipulate beneficial

42 plant microbiota to promote host colonization. Here, we show that the fungal pathogen Verticillium

43 dahliae has co-opted an ancient antimicrobial protein, that likely served in microbial competition in

44 terrestrial environments before land plants existed, as effector for the manipulation of fungal

45 competitors during host colonization. Thus, we demonstrate that pathogen effector repertoires

46 comprise antifungal proteins, and speculate such effectors could be exploited for the development

47 of novel antimycotics. 


\section{INTRODUCTION}

Microbes are found in a wide diversity of niches on our planet. To facilitate establishment within microbial communities, microbes secrete a multitude of molecules to manipulate each other. Many of these molecules exert antimicrobial activities and are exploited to directly suppress microbial coinhabitants in order to outcompete them for the limitedly available nutrients and space of a niche. Microbially-secreted antimicrobials encompass diverse molecules including peptides (AMPs) and lytic enzymes, but also non-proteinaceous molecules such as secondary metabolites. Soils are among the most biologically diverse and microbially competitive environments on earth. Microbial proliferation in the soil environment is generally limited by the availability of organic carbon (1), for which soil microbes continuously compete. Consequently, numerous saprophytic soil-dwelling microbes secrete potent antimicrobials that promote niche protection or colonization. Notably, these microbes are the primary source of our clinically used antibiotics $(2,3)$.

Like free-living microbes, also microbial plant pathogens secrete a multitude of molecules into their environment to mediate niche colonization $(4,5)$. The study of molecules secreted by microbial plant pathogens has been largely confined to the context of binary interactions between pathogens and hosts. To establish disease, plant pathogenic microbes secrete a plethora of so-called effectors, molecules of various kinds that promote host colonization and that are typically thought to mainly deregulate host immune responses $(4,6,7)$. Upon host colonization, plant pathogens encounter a plethora of plant-associated microbes that collectively form the plant microbiota, which represents a key factor for plant health. Beneficial plant-associated microbes are found in and on all organs of the plant and help to mitigate (a)biotic stresses (8-13). Plants shape their microbiota and specifically attract beneficial microbes to suppress pathogens (14-16). Hence, the plant microbiome can be considered an inherent, exogenous layer that complements the plant's endogenous innate immune system. We previously hypothesized that plant pathogens not only utilize effectors to target components of host immunity as well as other aspects of host physiology to support host colonization, but also to target the host microbiota in order to establish niche colonization $(4,5)$. We 
74 recently provided experimental evidence for this hypothesis by showing that the ubiquitously

75 expressed effector VdAve1 that is secreted by the soil-borne fungal plant pathogen Verticillium

76 dahliae acts as a bactericidal protein that promotes host colonization through the selective

77 manipulation of host microbiomes by suppressing microbial antagonists $(17,18)$. Additionally, we demonstrated that VdAve1 and a further antibacterial effector named VdAMP2 are exploited by $V$. dahliae for microbial competition in soil and promote virulence of $V$. dahliae in an indirect manner (18). Collectively, these observations demonstrate that $V$. dahliae dedicates part of its effector catalog towards microbiota manipulation. Likely, the $V$. dahliae genome encodes further effectors 82 that act in microbiome manipulation. consequence, fungal pathogen effectors involved in the manipulation of (host-associated) microbial communities may have evolved from ancestors that served in microbial competition in terrestrial niches hundreds of millions of years ago prior to land plant evolution. However, evidence for this hypothesis is presently lacking. of plant species (19). The fungus survives in the soil in the form of multicellular melanized resting structures, called microsclerotia, that offer protection against (a)biotic stresses and can persist in the soil for many years (20). Microsclerotia represent the major inoculum source of $V$. dahliae in nature

92 and their germination is triggered by carbon- and nitrogen-rich exudates from plant roots (21).

93 Following microsclerotia germination, fungal hyphae grow through the soil and rhizosphere towards

94 the roots of host plants. Next, $V$. dahliae colonizes the root cortex and crosses the endodermis, from

95 which it invades xylem vessels. Once the fungus enters those vessels it forms conidiospores that are

96 transported with the water flow until they get trapped, for instance by vessel end walls. This triggers

97 germination of the conidiospores, followed by penetration of cell walls, hyphal growth and renewed 98 sporulation, leading to systematic colonization of the plant (22). Once tissue necrosis commences 
100 when it emerges from the xylem vessels to invade adjacent host tissues, which is accompanied by

101 the production of microsclerotia. Upon littering and decomposition of plant tissues, these

102 microsclerotia are released into the soil (23).

103

104 RESULTS

105 To identify effectors potentially acting in microbiome manipulation, we recently queried the $V$.

106 dahliae secretome for structural homologs of known antimicrobial proteins (AMPs), which led to the

107 identification of ten candidates, including the functionally characterized VdAMP2 (18). Among the

108 remaining nine candidates we now identified a small cysteine-rich protein of $\sim 4.9 \mathrm{kDa}$, which we

109 name VdAMP3. As a first step in the characterization of VdAMP3 we assessed its predicted structure.

110 Interestingly, VdAMP3 is predicted to adopt a Cysteine-stabilized $\alpha \beta(\operatorname{CS} \alpha \beta)$ fold that is also found in

111 defensin-like proteins (Fig. 1a)(24-26). CS $\alpha \beta$ defensins represent a wide-spread and well-

112 characterized family of antimicrobial proteins that are presumed to share a single ancient origin in

113 the last common ancestor of animals, plants and fungi that produce these proteins today (24-27). It

114 is important to note, however, that many typical small cysteine-rich pathogen effectors adopt AMP-

115 like confirmations, and that tertiary structures of several AMP families strongly resemble each other

$116(27,28)$. Hence, structure prediction can easily lead to false-positive classifications as AMP or

117 allocation to the wrong AMP family.

$118 \quad \operatorname{CS} \alpha \beta$ defensins, or so-called cis-defensins, owe their structure to highly conserved cis-

119 orientated disulfide bonds that establish an interaction between a double- or triple-stranded

120 antiparallel $\beta$-sheet with an $\alpha$-helix $(25,27)$. To validate the prediction of VdAMP3 as a member of

121 this ancient antimicrobial protein family, we aligned its amino acid sequence with the antibacterial

$122 \mathrm{CS} \alpha \beta$ defensins Plectasin and Eurocin, from the saprophytic Ascomycete species Pseudoplectania

123 nigrella and Eurotium amstelodami (formerly Aspergillus amstelodami), respectively (29-31).

124 Although the biological relevance of these defensins for the respective fungi remains unclear, their

125 antibacterial activity and protein structure have been well characterized, which lead to their 
126 recognition as genuine $\operatorname{CS} \alpha \beta$ defensins (29-31). Although the overall identity between the three

127 proteins was rather low (25-40\%), protein sequence alignment revealed that VdAMP3 contains the

128 six highly conserved cysteine residues that are considered crucial for the structure of CS $\alpha \beta$ defensins

129 (Fig. 1b)(27). To further substantiate the emerging picture that VdAMP3 belongs to this particular

130 protein family, and that the detected similarities with Plectasin and Eurocin are not the result of

131 convergent protein evolution, we queried the predicted proteomes of the fungi from the JGI 1000

132 Fungal Genomes Project (32) for homologs of VdAMP3 with higher sequence identity and included a

133 subset of those in the protein alignment (Fig. 1C). Interestingly, besides homologs in Ascomycota and

134 Basidiomycota, our sequence similarity search also revealed homologs in early-diverging fungi from

135 the subphyla Mucoromycotina and Zoopagomycota (both formerly classified as Zygomycota (33))

136 (Fig. 1c). Importantly, this divergence is estimated to have taken place approximately 900 million

137 years ago (34), indicating it preceded the evolution of the first land plants approximately 450 million

138 years later (34-37). Consequently, this analysis indicates that VdAMP3 evolved from an ancestral

139 fungal gene hundreds of millions of years ago, before land plants existed. 


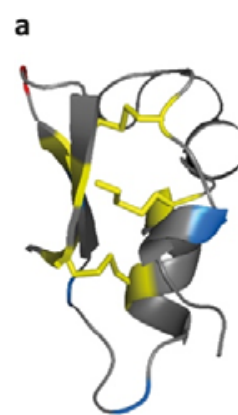

VdAMP3

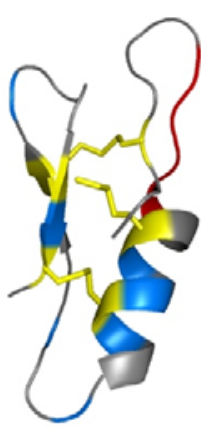

Plectasin b

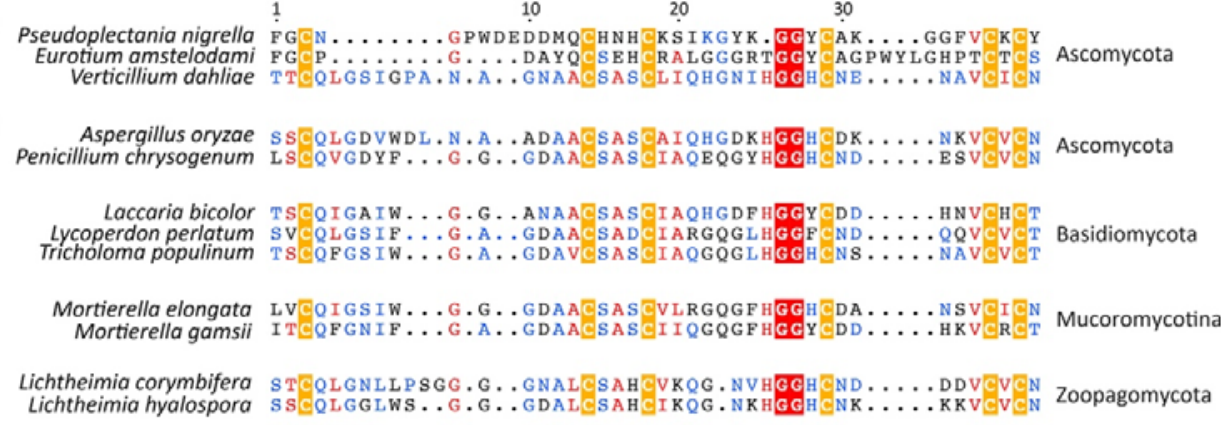

141 Figure 1 . The $V$. dahliae effector VdAMP3 evolved from an ancient fungal protein. (a) VdAMP3 142 (left) is predicted to adopt a cysteine-stabilized $\alpha \beta(\mathrm{CS} \alpha \beta)$ defensin-like fold. The structure of the $143 \mathrm{CS} \alpha \beta$ defensin Plectasin (right) of the fungus Pseudoplectania nigrella is included as reference. The 144 disulfide bonds stabilizing the antiparallel $\beta$-sheets and the $\alpha$-helix are highlighted in yellow. 145 Positively and negatively charged amino acid residues are highlighted in blue and red, respectively. 146 (b) Protein sequence alignment with $\mathrm{CS} \alpha \beta$ defensins Plectasin and Eurocin (Eurotium amstelodami) 147 supports the structure prediction of VdAMP3. (c) VdAMP3 homologs are widespread in the fungal 148 kingdom. Protein sequence alignment of VdAMP3 with a subset of its homologs identified in higher 149 (Ascomycota and Basidiomycota) and lower fungi (Mucoromycotina and Zoopagomycota). The 150 alignment as shown in (b-c) displays the most conserved region of the CS $\alpha \beta$ defensin protein family 151 and was performed using HMMER and visualized with Espript3. The highly conserved cysteine and 152 glycine residues that contribute to the $\operatorname{CS} \alpha \beta$ defensin structure are highlighted by yellow and red 153 backgrounds, respectively. The homologs displayed in (c) were identified using blastP in the 154 predicted proteomes of the respective fungi included in the JGI 1000 Fungal Genomes Project (32).

156 As a first step to determine the role of VdAMP3 in $V$. dahliae infection biology, we assessed 157 conditions for $V d A M P 3$ expression. Transcriptome analysis of diverse $V$. dahliae strains during 158 colonization of a diversity of hosts did not reveal in planta expression of VdAMP3 thus far (17, 38-

159 40). However, strong induction of this effector gene was reported during microsclerotia formation in 160 a transcriptome analysis of $V$. dahliae strain XS11 grown in vitro (24). To validate this finding, we 161 analyzed in vitro expression of $V d A M P 3$ in $V$. dahliae strain JR2. To this end, $V$. dahliae conidiospores 162 were spread on nitrocellulose membranes placed on top of solid minimal medium and fungal 163 material was harvested prior to microsclerotia formation, after 48 hours of incubation, and after the 164 onset of microsclerotia formation, after 96 hours of incubation. Expression of VdAMP3 was 165 determined at both time points with real-time PCR alongside expression of the Chr6g02430 gene 166 that encodes a putative cytochrome P450 enzyme that acts as a marker for microsclerotia formation 
167 (24, 41). Consistent with the observations for V. dahliae strain XS11 (24), no VdAMP3 expression was

168 detected at 48 hours when also Chr6g02430 was not expressed and no visual microsclerotia

169 formation could be observed on the growth medium (Fig. 2a). However, induction of VdAMP3 as

170 well as Chr6g02430 was observed after 96 hours of incubation, at which time point also the

171 formation of microsclerotia on the growth medium became apparent (Fig. 2a). Collectively these

172 data demonstrate that expression of VdAMP3 coincides with microsclerotia formation in vitro also

173 for V. dahliae strain JR2.

174 Although previous transcriptome analyses failed to detect in planta expression of VdAMP3,

175 we realized that these analyses were predominantly performed for infection stages when the fungus

176 is still confined to the xylem vessels and microsclerotia formation had not yet been initiated.

177 Accordingly, in planta expression of VdAMP3 may have been missed. Thus, we inoculated Nicotiana

178 benthamiana with $V$. dahliae and determined expression of VdAMP3 in leaves and petioles sampled

179 at different time points and displaying different disease phenotypes, ranging from asymptomatic at

180 seven days post inoculation (dpi) to complete necrosis at $22 \mathrm{dpi}$. As expected, a strong induction of

181 the previously characterized VdAve1 effector gene was detected at seven and $14 \mathrm{dpi}$ (Fig. 2b) (17,

182 18). In contrast, however, no expression of $V d A M P 3$ was recorded, even the latest time point

183 when the leaf tissue had become completely necrotic (Fig. 2b). Importantly, also no Chr6g02430

184 expression was detected at any of these time points (Fig. 2b), suggesting that microsclerotia

185 formation had not yet started in these tissues. Indeed, visual inspection of the necrotic plant tissue

186 collected at $22 \mathrm{dpi}$ did not reveal microsclerotia presence. To induce microsclerotia formation, $V$.

187 dahliae-inoculated $N$. benthamiana plants harvested at $22 \mathrm{dpi}$ were sealed in plastic bags and

188 incubated in the dark to increase the relative humidity and mimic conditions that occur during tissue

189 decomposition in the soil. Interestingly, after eight days of incubation the first microsclerotia could

190 be observed, and induction of VdAMP3 as well as Chr6g02430 was detected (Fig. 2c). Collectively,

191 these findings suggest that in planta expression of VdAMP3 coincides with microsclerotia formation, 
bioRxiv preprint doi: https://doi.org/10.1101/2021.06.10.447847; this version posted June 10, 2021. The copyright holder for this preprint (which was not certified by peer review) is the author/funder, who has granted bioRxiv a license to display the preprint in perpetuity. It is made available under aCC-BY 4.0 International license.

a

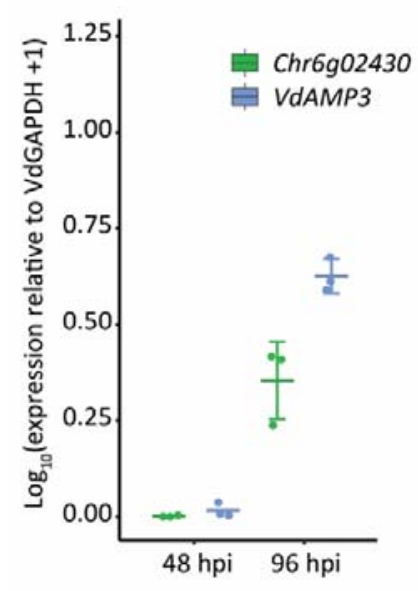

b

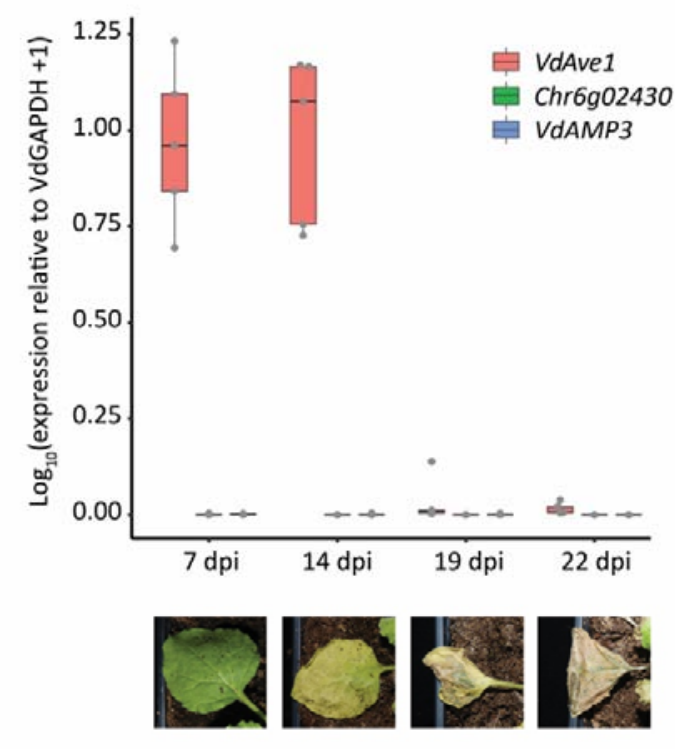

Single leaf

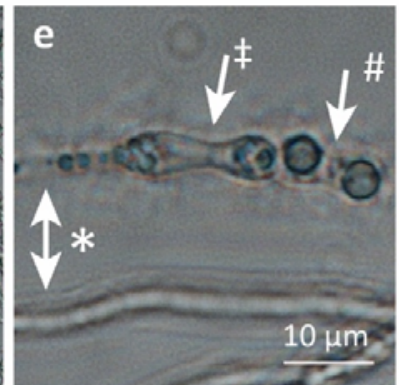

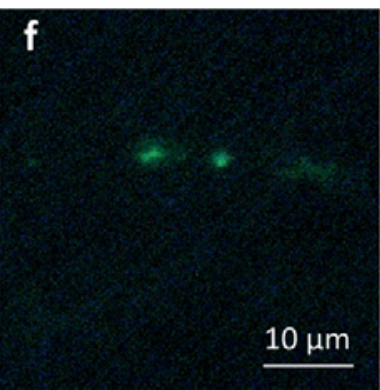

194

195

196

197

198

199

200

201

202

203

204

205

206

207
Figure 2. VdAMP3 is specifically expressed in hyphal cells that develop into microsclerotia. (a) Expression of VdAMP3 and the marker gene for microsclerotia development Chr6g02430, relative to the household gene $V d G A P D H$ at 48 and 96 hours of in vitro cultivation ( $\mathrm{N}=3$ ). (b) Expression of VdAve1, VdAMP3 and Chr6g02430 in N. benthamiana leaves from 7 to 22 days post inoculation (dpi) $(\mathrm{N}=5)$. (c) Expression of $V d A v e 1, V d A M P 3$ and Chr6g02430 in tissue of $N$. benthamiana plants harvested at 22 days post inoculation after 8 days of incubation in sealed plastic bags ( $N=3$ ). (d) Microsclerotia formation of a pVdAMP3::eGFP reporter mutant as detected after 7 days of cultivation in Czapek Dox medium. Typical chains of microsclerotia $(42,43)$ are indicated by arrows. (e) Bright field image of various $V$. dahliae cell types after 7 days of cultivation in Czapek Dox, including hyphae $(*)$, swollen hyphal cells developing into microsclerotia ( $¥$ ) and mature microsclerotia cells (\#). (f) GFP signal for the image as shown in (e), indicative for activity of the VdAMP3 promoter, is exclusively detected in the swollen hyphal cells developing into microsclerotia.

(g) Overlay of (e) and (f). 
208 To determine more precisely where VdAMP3 is expressed, and to improve our understanding of how

209 V. dahliae may benefit from effector expression during microsclerotia formation, we generated a $V$.

210 dahliae reporter strain expressing eGFP under control of the VdAMP3 promoter. Intriguingly,

211 microscopic analysis of the reporter strain during microsclerotia formation stages in vitro (Fig. 2d),

212 revealed that $V d A M P 3$ is expressed by swollen hyphal cells that act as primordia that subsequently

213 develop into microsclerotia, but not by the adjacent hyphal cells or recently developed

214 microsclerotia cells (Fig. 2e-g). This highly specific expression of VdAMP3 suggests that the effector

215 protein may facilitate the formation of microsclerotia in decaying host tissue. Given its presumed

216 antimicrobial activity, VdAMP3 may be involved in antagonistic activity against opportunistic decay

217 organisms in this microbially competitive niche.

218 To determine if VdAMP3 indeed exerts antimicrobial activity, we incubated a randomly

219 selected panel of bacterial isolates with the effector protein and monitored their growth in vitro.

220 VdAMP3 concentrations as high as $20 \mu \mathrm{M}$ resulted in no or only marginal bacterial growth inhibition

221 (Supplementary Fig. 1). A similar assay with fungal isolates showed that incubation with $5 \mu \mathrm{M}$ of

222 VdAMP3 already markedly affected growth of the filamentous fungi Alternaria brassicicola and

223 Cladosporium cucumerinum and the yeasts Pichia pastoris and Saccharomyces cerevisiae (Figure 3a).

224 This finding suggests that VdAMP3 displays more potent activity against fungi than against bacteria. 
bioRxiv preprint doi: https://doi.org/10.1101/2021.06.10.447847; this version posted June 10, 2021. The copyright holder for this preprint (which was not certified by peer review) is the author/funder, who has granted bioRxiv a license to display the preprint in perpetuity. It is made available under aCC-BY 4.0 International license.

a

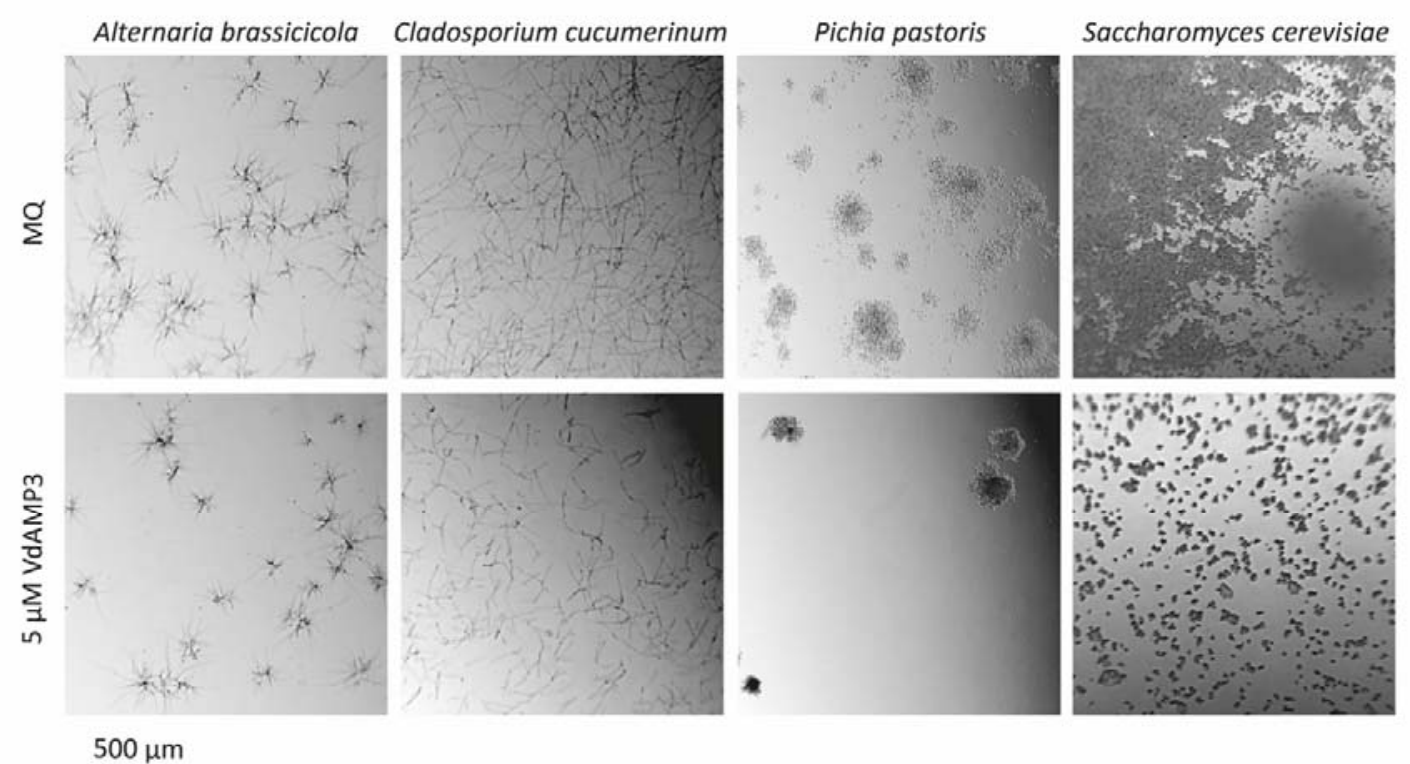

b

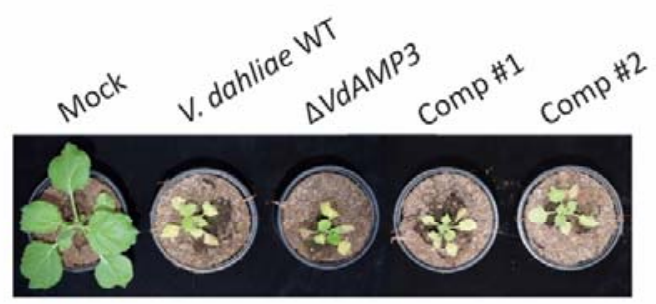

C

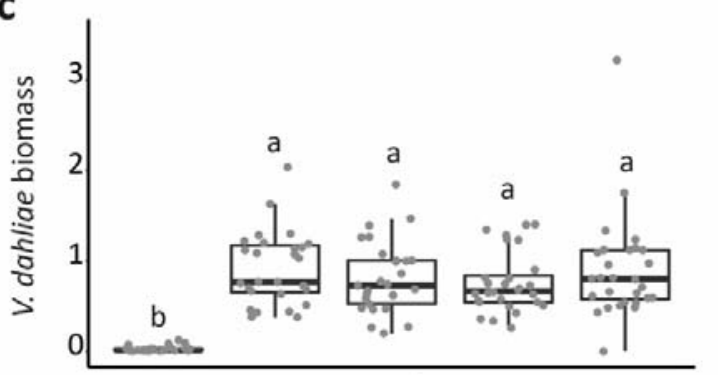

$14 \mathrm{dpi}$ d

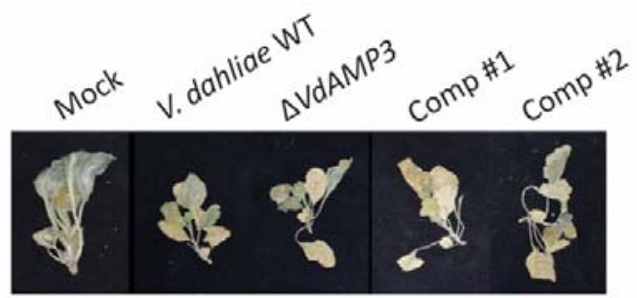

e

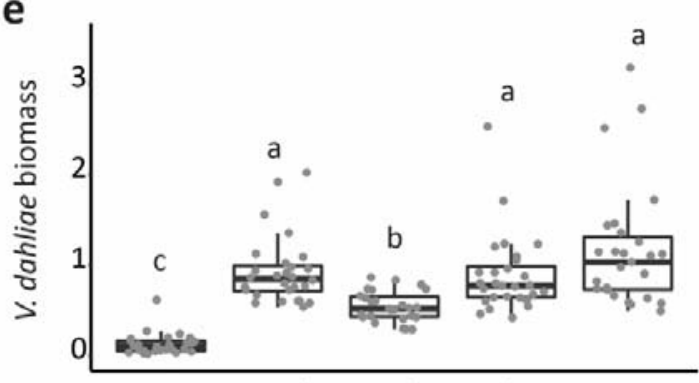

14 dpi +28 days in bag

225 Figure 3. VdAMP3 is an antifungal protein that contributes to $V$. dahliae biomass accumulation in

226 the decaying host phyllosphere. (a) Microscopic pictures of fungal isolates grown in $0.05 \mathrm{x}$ potato

227 dextrose broth supplemented with $5 \mu \mathrm{M}$ VdAMP3 or ultrapure water (MQ). VdAMP3 impairs growth

228 of Alternaria brassicicola, Cladosporium cucumerinum, Pichia pastoris and Saccharomyces cerevisiae.

229 Pictures were taken after 24 (A. brassicicola, C. cucumerinum and S. cerevisiae) or 64 (P. pastoris)

230 hours of incubation. (b) VdAMP3 does not contribute to establishment of Verticillium wilt disease in

$231 N$. benthamiana. Photos display representative phenotypes of $N$. benthamiana plants infected by

232 wild-type $V$. dahliae (WT), the VdAMP3 deletion ( $\triangle V$ dAMP3) and two complementation (Comp)

233 mutants 14 days post inoculation. (c) Relative $V$. dahliae biomass in above-ground $N$. benthamiana

234 tissues determined with real-time PCR. Different letter labels represent significant differences (one-

235 way ANOVA and Tukey's post-hoc test; $\mathrm{p}<0.05 ; \mathrm{N} \geq 27$ (d) Representative phenotypes of $N$.

236 benthamiana plants as shown in (b) after 28 days of incubation in plastic bags. (e) Relative $V$. dahliae

237 biomass in $N$. benthamiana tissues as displayed in (d). Letters represent significant differences (one-

238 way ANOVA and Tukey's post-hoc test; $p<0.05 ; \mathrm{N} \geq 27$ ). 
239 To study the importance of the antifungal activity of VdAMP3 during and after host

240 colonization, a VdAMP3 deletion mutant was generated as well as complementation strains

241 (Supplementary Fig 2). Importantly, targeted deletion of VdAMP3 did not affect growth nor

242 microsclerotia formation in vitro (Supplementary Fig. 3a,b). To determine if VdAMP3 contributes to

243 Verticillium wilt disease development, N. benthamiana plants were inoculated with wild-type $V$.

244 dahliae and the VdAMP3 deletion mutant. In line with our inability to detect expression during early

245 infection stages, disease phenotypes and $V$. dahliae biomass quantification using real-time PCR did

246 not reveal a contribution of VdAMP3 to host colonization up to two weeks after inoculation (Fig.

$2473 b, c)$. To test if VdAMP3 contributes to $V$. dahliae niche establishment following systemic host

248 colonization, we harvested the $N$. benthamiana plants and sealed them in plastic bags to induce

249 microsclerotia formation. Interestingly, following four weeks of incubation, $V$. dahliae biomass

250 quantification in $N$. benthamiana plants inoculated with the various genotypes using real-time PCR

251 revealed a significant reduction in biomass of the VdAMP3 deletion mutant when compared with

252 wild-type $V$. dahliae and complementation mutants (Fig 3d,e).

253 To investigate if the effects of VdAMP3 are limited to $N$. benthamiana, or whether those also

254 extend to other hosts, we inoculated Arabidopsis thaliana plants with wild-type $V$. dahliae and the

255 VdAMP3 deletion mutant. Consistent with our observations for $N$. benthamiana, deletion of

256 VdAMP3 did not affect establishment of Verticillium wilt in A. thaliana (Supplementary Fig. 4a,b).

257 However, $V$. dahliae biomass quantification in above-ground $A$. thaliana tissues at three weeks post

258 inoculation revealed reduced accumulation of $V$. dahliae in the absence of VdAMP3 (Supplementary

259 Fig. 4c). Thus, the effects of VdAMP3 are not restricted to a single host.

260 As in vitro antimicrobial activity assays pointed towards fungi as the primary targets of

261 VdAMP3, we speculated that $V$. dahliae exploits VdAMP3 to suppress fungal competitors in

262 decomposing host tissues to safeguard the formation of its resisting structures. To characterize the

263 microbiota associated with N. benthamiana decomposition and to determine the impact of VdAMP3

264 on these microbial communities, we characterized the phyllosphere microbiota of fresh mock- 
265 inoculated $N$. benthamiana plants, and decaying plants diseased by $V$. dahliae WT or the VdAMP3

266 deletion mutant incubated in plastic bags, through shotgun metagenomic sequencing. Consistent

267 with a primary role for fungi in the decomposition of dead plant material (44-48) we detected a

268 significant increase of fungi and decrease of bacteria in the phyllosphere of the $N$. benthamiana

269 plants diseased by the V. dahliae strains when compared with healthy mock-treated plants (Fig. 4a-

270 b). These changes are accompanied by a reduced alpha diversity in the decaying phyllospheres (Fig.

271 4c). Additionally, principal coordinate analysis (PCoA) based on Bray-Curtis dissimilarities (beta

272 diversity) uncovered clear separation of the microbiota of the healthy plants from those in decay

273 (Fig. 4d). The PCoA also revealed a weaker, yet potentially relevant, separation of the microbiota

274 colonized by $V$. dahliae WT and the VdAMP3 deletion mutant, which suggests that secretion of

275 VdAMP3 manipulates microbiome compositions (Fig. 4d). Intriguingly, when we compared the

276 abundances of the identified microbial genera between the microbiomes colonized by $V$. dahliae WT

277 and the VdAMP3 deletion mutant, we detected significantly more differentially abundant fungi

278 (10.1\%) than bacteria (3.8\%) (Fig. 4e) (Supplementary Table 1-2). Interestingly, whereas the number

279 of bacterial genera that display an increased or a decreased abundance in the presence of VdAMP3

280 is more or less equal, the vast majority of the differentially abundant fungal genera (82.1\%) are

281 repressed in the presence of VdAMP3 (Fig. 4f). Moreover, while no consistent suppression of

282 bacterial genera from the same class could be detected, we exclusively identified suppression of the

283 differentially abundant fungal genera from the Saccharomycetes or Sordariomycetes in the presence

284 of VdAMP3 (Fig. 4g-h). Thus, these observations indicate that V. dahliae VdAMP3 mainly acts as an

285 antifungal effector protein that displays selective activity that predominantly impacts the 286 mycobiome in the decaying host phyllosphere. 
bioRxiv preprint doi: https://doi.org/10.1101/2021.06.10.447847; this version posted June 10, 2021. The copyright holder for this preprint (which was not certified by peer review) is the author/funder, who has granted bioRxiv a license to display the preprint in perpetuity. It is made available under aCC-BY 4.0 International license.

287

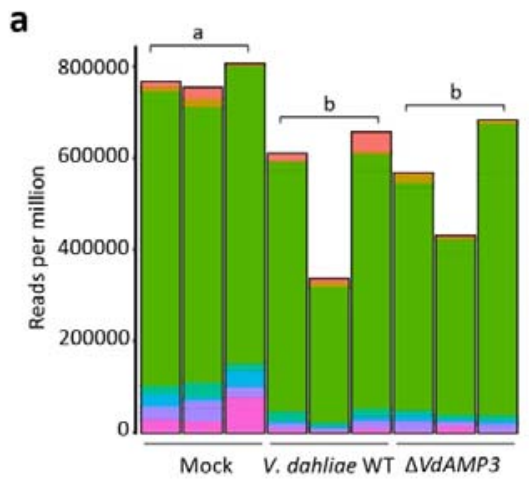

C

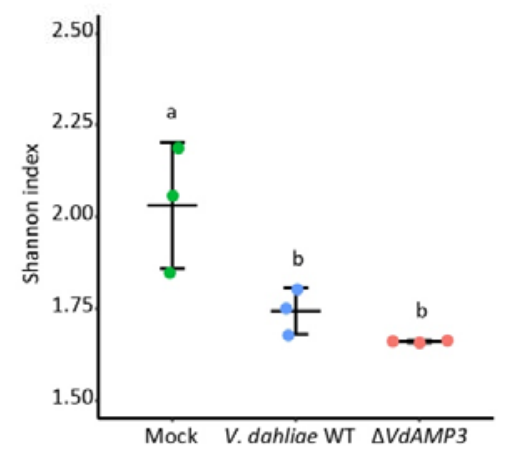

g

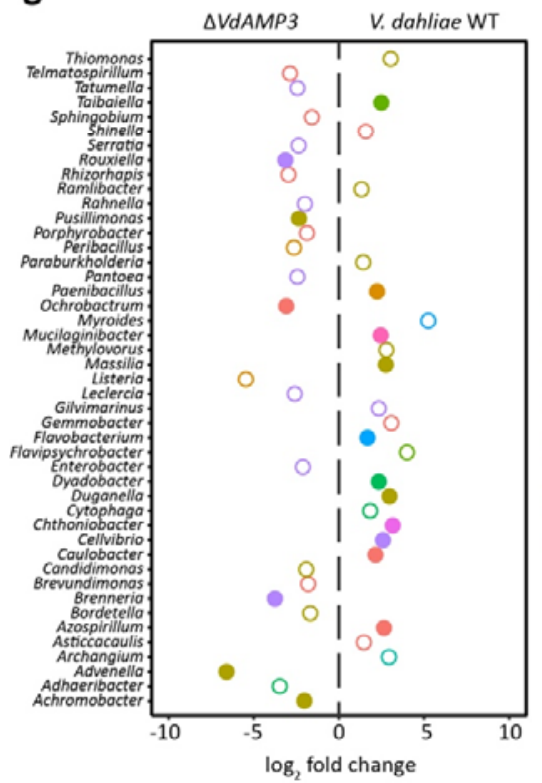

b

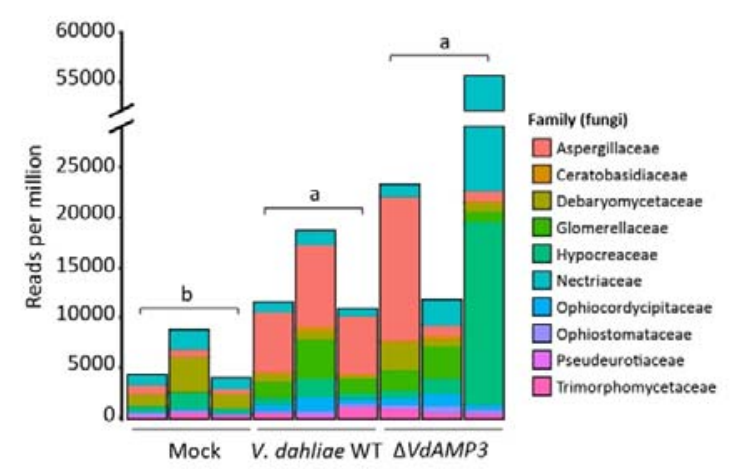

d

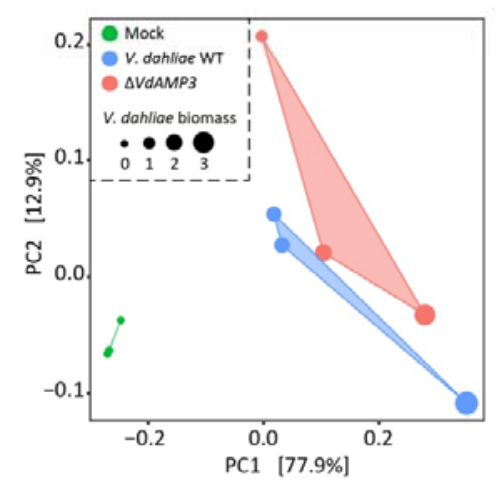

e
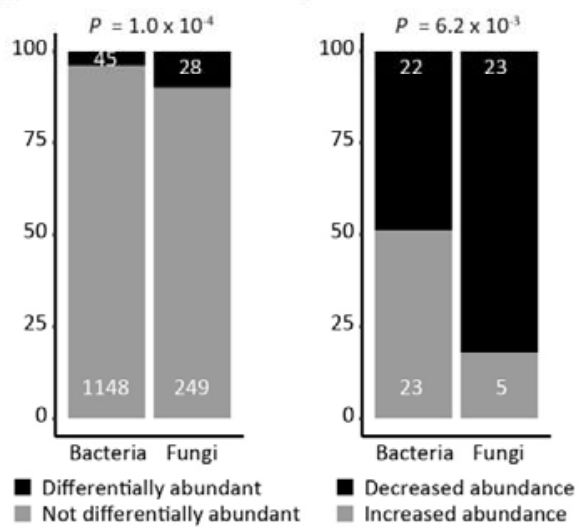

Class (bacteria)

Alphaproteobacteria

Bacilli

Betaproteobacteria

Chitinophagia

Cytophagia

Deltaproteobacteria

- Erysipelotrichia

Flavobacteriia

Gammaproteobacteria

Spartobacteria

Sphingobacteriia

$P$ adjusted $<0.05$
$P<0.05$ h

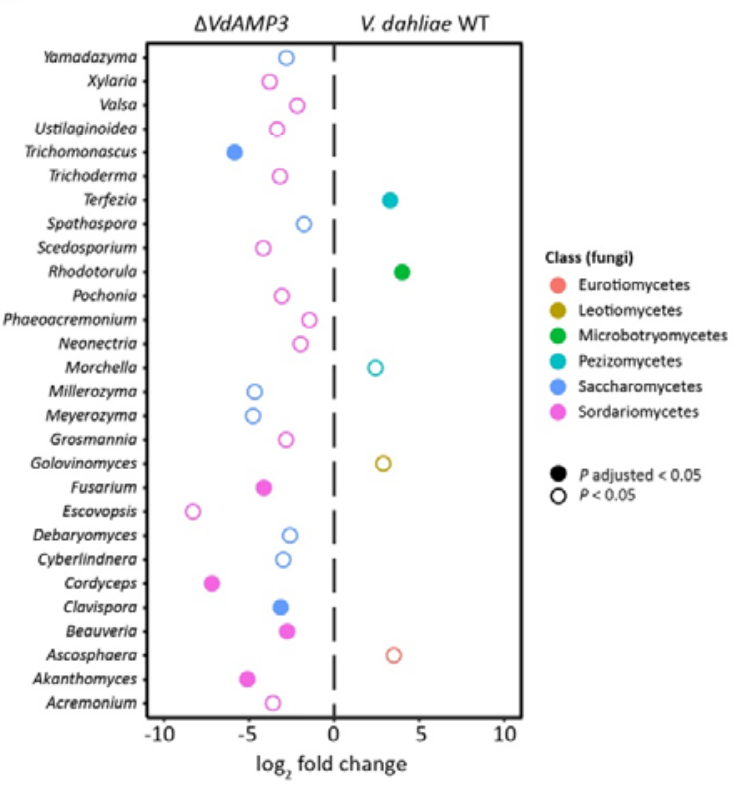

289 Figure 4. VdAMP3 manipulates the mycobiome of the decaying $\boldsymbol{N}$. benthamiana phyllosphere. (a-

290 b) $V$. dahliae-induced decay of the $N$. benthamiana phyllosphere is associated with a decreased 291 bacterial, and increased fungal, abundance. Relative abundance of bacteria (a) and fungi (b), 292 excluding $V$. dahliae, in the phyllosphere of decaying $N$. benthamiana plants colonized by wild-type 
293 V. dahliae or the VdAMP3 deletion mutant (14 days post inoculation and after 28 days of incubation

294 in plastic bags), and in the phyllosphere of fresh $N$. benthamiana plants (mock). Letters represent

295 significant differences in total bacterial/fungal abundance between the three treatments (one-way

296 ANOVA and Tukey's post hoc test; $P$ ? $<<0.05 ; \mathrm{N}=3$ ). (c) $V$. dahliae-induced decay of $N$. benthamiana

297 plants impacts alpha diversity of the phyllosphere. The plot displays the average Shannon index \pm

298 SD, letters represent significant differences (one-way ANOVA and Tukey's post hoc test; $P$ [?] $<0.05$;

$299 \mathrm{~N}=3$ ). (d) Principal coordinate analysis based on Bray-Curtis dissimilarities (beta diversity) reveals

300 separation of the microbiomes based on the three different treatments. (e) Differential abundance

301 analysis of microbial genera between the microbiomes colonized by V. dahliae WT and the VdAMP3

302 deletion mutant indicates that secretion of VdAMP3 significantly impacts a larger proportion of the

303 fungi than of the bacteria (two-tailed Fisher's exact test). (f) Of the differentially abundant microbial

304 genera, significantly more fungi display a decreased abundance in the presence of VdAMP3 when

305 compared with the bacteria (two-tailed Fisher's exact test). (g-h) Overview of the differentially

306 abundant bacterial ( $\mathrm{g}$ ) and fungal (h) genera. The plots display increased (positive $\log _{2}$ fold change)

307 or decreased (negative $\log _{2}$ fold change) abundance in the presence of $V$. dahliae WT when

308 compared with the VdAMP3 deletion mutant (Wald test, $P$ adjusted $<0.05$ and $P<0.05, \mathrm{~N}=3$ ).

309 Differentially abundant fungal genera from the Saccharomycetes or Sordariomycetes are

310 consistently suppressed in the presence of VdAMP3.

312 To further substantiate that the suppression of the Saccharomycetes and Sordariomycetes is a direct

313 consequence of the VdAMP3 activity, we incubated fungal species belonging to the suppressed

314 genera with the effector to determine their sensitivity. In line with the previously observed

315 sensitivity of the Saccharomycetes $P$. pastoris and S. cerevisiae, also the Saccharomycete species

316 Cyberlindnera jadinii, Debaryomyces vanrijiae, Rhodotorula bogoriensis and Meyerozyma amylolytica

317 displayed markedly reduced growth in the presence of VdAMP3 (Fig. 5a, Supplementary Fig. 5).

318 Similarly, growth of the Sordariomycetes Cordyceps militaris and Trichoderma viride was inhibited by

319 the effector (Supplementary Fig. 5). Hence, these findings support the observed suppression of the

320 Saccharomycetes and Sordariomycetes in the $\mathrm{N}$. benthamiana phyllosphere mycobiome as a direct

321 consequence of VdAMP3 activity.

322 The cell type-specific expression of VdAMP3, combined with its role in mycobiome

323 manipulation, strongly suggests that VdAMP3 is exploited to ward off fungal niche competitors in

324 planta to safeguard the formation of $V$. dahliae microsclerotia. To test if VdAMP3 indeed is essential

325 for $V$. dahliae microsclerotia formation in the presence of other fungi, we co-cultivated $V$. dahliae

326 WT and the VdAMP3 deletion mutant with D. vanrijiae and M. amylolytica. Once microsclerotia 
bioRxiv preprint doi: https://doi.org/10.1101/2021.06.10.447847; this version posted June 10, 2021. The copyright holder for this preprint (which was not certified by peer review) is the author/funder, who has granted bioRxiv a license to display the preprint in perpetuity. It is made available under aCC-BY 4.0 International license.

327 formation by $V$. dahliae WT became apparent (Fig. 5b), we quantified the number of resting

328 structures that were formed when compared with the VdAMP3 deletion mutant. As anticipated, we

329 detected a significant reduction of microsclerotia formed by the VdAMP3 deletion mutant in the

330 presence of both fungal species, confirming that $V$. dahliae relies on the antifungal activity of

331 VdAMP3 to form microsclerotia in the presence of particular fungal niche competitors (Fig. 5c,d).

a

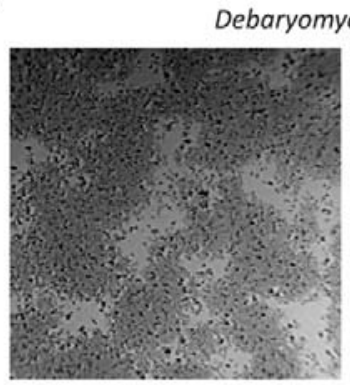

$\mathrm{MQ}$

b
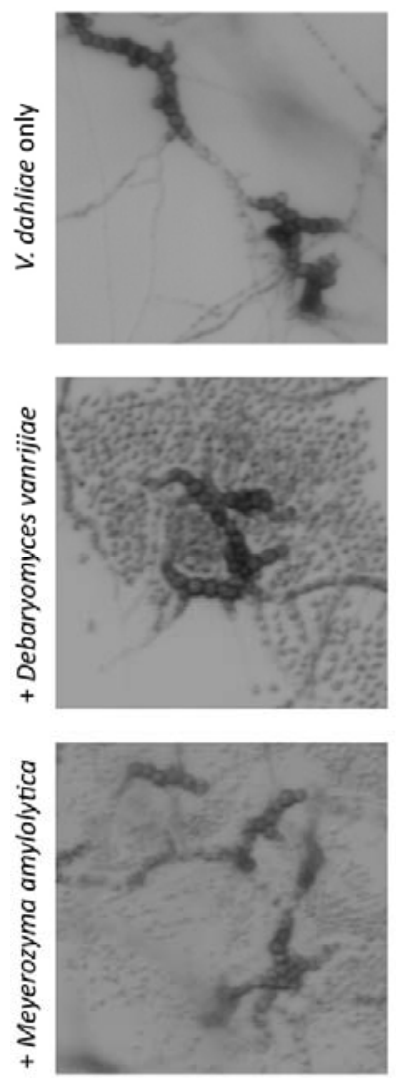

$100 \mu \mathrm{m}$
C
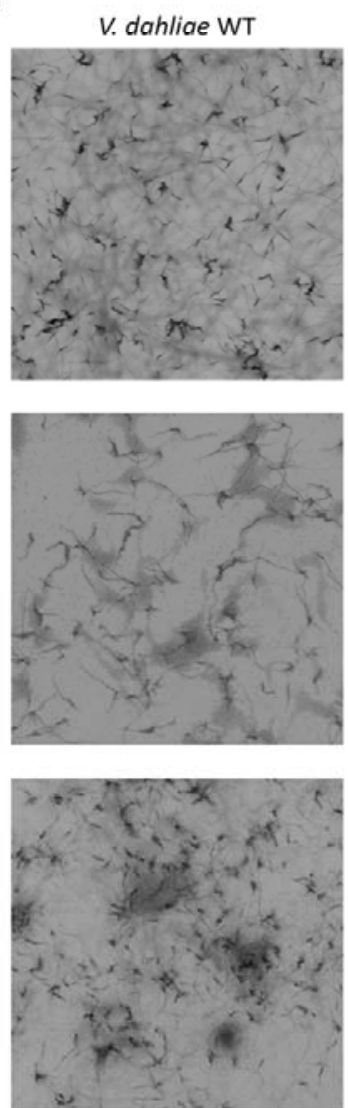

$500 \mu \mathrm{m}$

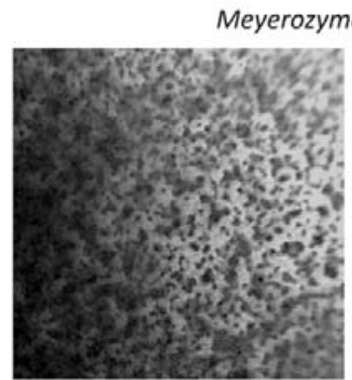

$\mathrm{MQ}$
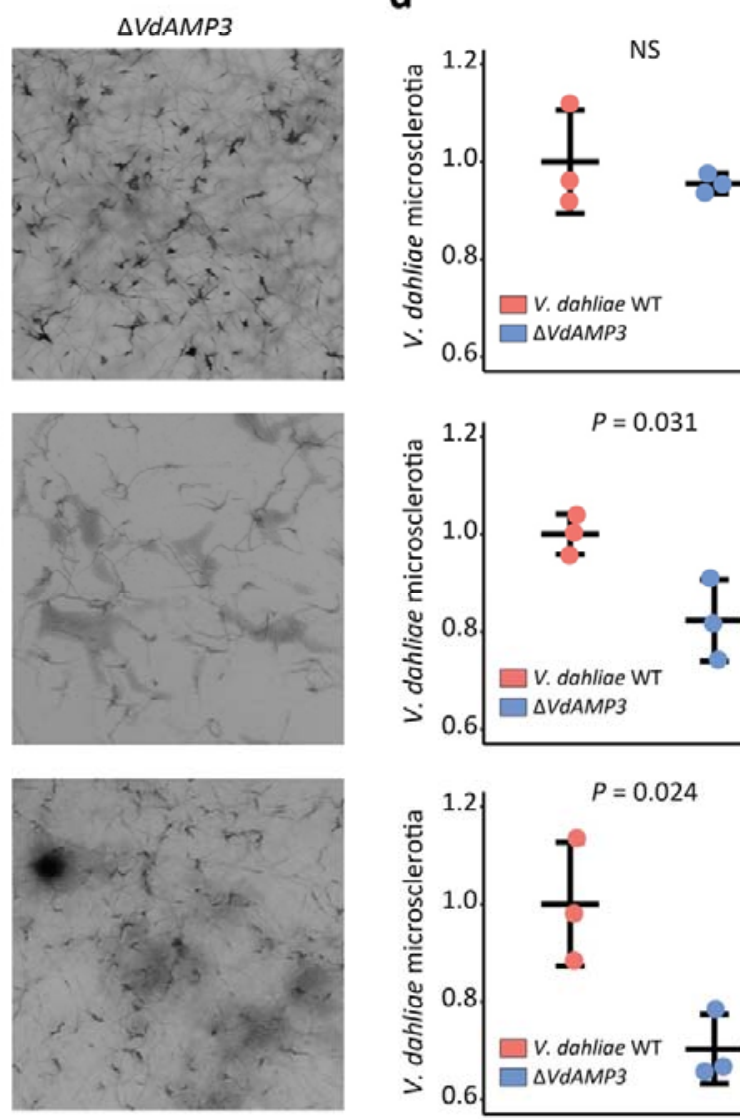
334 Figure 5. VdAMP3 contributes to $\mathbf{V}$. dahliae microsclerotia formation in the presence of fungal

335 niche competitors. (a) Debaryomyces vanrijae and Meyerozyma amylolytica are inhibited by 336 VdAMP3. Microscopic pictures of the fungal species grown in $0.05 x$ potato dextrose broth 337 supplemented with $5 \mu \mathrm{M}$ VdAMP3 or ultrapure water (MQ). Pictures were taken after $10(D$. 338 vanrijae) or 24 (Meyerozyma amylolytica) hours of cultivation. (b) Close-up of V. dahliae 339 microsclerotia formed after seven days of cultivation in the presence of $D$. vanrijae or $M$. 340 amylolytica. (c) VdAMP3 contributes to $V$. dahliae microsclerotia formation in the presence of the 341 other fungal species. Representative microscopic pictures displaying the co-culture of $V$. dahliae with 342 D. vanrijae or $M$. amylolytica. Pictures were taken after seven days of co-cultivation. (d) Relative 343 number of microsclerotia formed by $V$. dahliae WT and the VdAMP3 deletion mutant in the 344 presence of $D$. vanrijae or M. amylolytica as determined using Image (unpaired two-sided student's 345 t-test; $\mathrm{N}=3$ ). 


\section{DISCUSSION}

347 Microbes secrete a plethora of molecules to promote niche colonization (4). Free-living microbes are

348 well known producers of antimicrobials that are secreted to outcompete microbial co-inhabitants to

349 establish themselves in a microbial community. Microbial plant pathogens secrete a diversity of so-

350 called effector molecules during host ingress, many of which are small cysteine-rich proteins that

351 deregulate host immune responses to promote colonization $(4,6,7)$. While investigating the

352 vascular wilt fungus $V$. dahliae, we recently demonstrated that plant pathogens not only exploit

353 effector proteins to promote disease establishment through direct host manipulation, but also

354 through the manipulation of plant microbiota by means of antibacterial activities (18). Considering

355 that the advent of fungi on earth preceded land plant evolution, we speculated that a subset of the

356 pathogen effectors involved in host microbiota manipulation may have evolved from antimicrobial

357 proteins that originally functioned in microbial competition in terrestrial niches before the first land

358 plants appeared and plant pathogenicity evolved. Here, we demonstrated that the soil-borne fungal

359 plant pathogen $V$. dahliae has co-opted an ancient antimicrobial protein as effector for mycobiome

360 manipulation in planta to safeguard the formation of its resting structures. Thus, our findings

361 indicate that plant pathogenicity in fungi is not exclusively associated with the evolution of novel

362 effectors that manipulate plants or their associated microbial communities, but also with the co-

363 option of previously evolved secreted proteins that initially served alternative lifestyles, such as

364 saprotrophism, as effectors to promote host colonization. Moreover, our findings indicate that

365 effector-mediated manipulation of plant microbiota by microbial plant pathogens is not confined to

366 bacterial targets, but extends to eukaryotic microbes.

367 Functional characterization of VdAMP3 unveiled that the effector evolved to play a life

368 stage-specific role in microbiome-manipulation during microsclerotia formation by $V$. dahliae.

369 Recently, we described the characterization of the first microbiome-manipulating effectors secreted

370 by V. dahliae; VdAve1 and VdAMP2 (18). VdAve1 is a ubiquitously expressed bactericidal effector

371 that promotes $V$. dahliae host colonization through the selective manipulation of host microbiota in 
372 the roots as well as in the xylem by suppressing microbial antagonists. Moreover, VdAve1 is also

373 expressed in the soil biome where it similarly contributes to niche colonization. Intriguingly, VdAMP2

374 is exclusively expressed in soil, and like VdAve1 exerts an antibacterial activity that contributes to

375 niche establishment. Interestingly, VdAMP2 and VdAve1 display divergent activity spectra, and

376 therefore likely complement each other for optimal soil colonization. In decaying host tissue neither

377 VdAve1 nor VdAMP2 is expressed, yet VdAMP3 expression occurs. Collectively, our findings for

378 VdAve1, VdAMP2 and VdAMP3 demonstrate that $V$. dahliae dedicates a substantial part of its

379 catalog of effector proteins towards microbiome manipulation, and that each of these effectors act

380 in a life stage-specific manner.

381 The life stage-specific exploitation of the in planta secreted antimicrobial effectors VdAve1

382 and VdAMP3 is well reflected by their antimicrobial activities and by the microbiota of the niches

383 where they act. Contrary to previous $V$. dahliae transcriptome analyses, that repeatedly identified

384 VdAve1 as one of the most highly expressed effector genes in planta (17, 38-40), we detected a

385 repression of the effector gene in decomposing $N$. benthamiana tissues (Fig. 1b,c). Characterization

386 of the antimicrobial activity exerted by $\mathrm{VdAve1}$ previously uncovered that the protein exclusively

387 affects bacteria and does not impact fungi (18). Thanks to their ability to produce a wide diversity of

388 hydrolytic enzymes, fungi are the primary decomposers of plant debris on earth (44). The

389 phyllosphere of plants comprises a diversity of fungi (49-51). Importantly, upon plant senescence,

390 these fungi are provided the first access to decaying material on which they can act opportunistically

391 once host immune responses have faded. Accordingly, we detected an increased abundance of fungi

392 in the phyllosphere of the decomposing $N$. benthamiana plants diseased by $V$. dahliae when

393 compared with healthy plants (Fig. 4b). The observed repression of VdAve1 and the subsequent

394 induction of VdAMP3 in a niche where $V$. dahliae encounters more fungal competition, underscores

395 the notion that $V$. dahliae tailors the expression of its microbiome-manipulating effectors according

396 to the various microbiota that it encounters during the different life stages. Along these lines it is

397 tempting to speculate that during saprotrophism in soil $V$. dahliae exploits antimicrobial effector 
398 proteins to ward off other eukaryotic competitors including soil-dwelling parasites such as

399 fungivorous nematodes or protists. However, evidence for this hypothesis is presently lacking.

400 Antimicrobial resistance in bacteria and fungi is posing an increasing threat to human health.

401 Possibly, microbiome-manipulating effectors represent a valuable source for the identification and

402 development of novel antimicrobials that can be deployed to treat microbial infections. Arguably,

403 our findings that microbiome-manipulating effectors secreted by plant pathogens also comprise

404 antifungal proteins opens up opportunities for the identification and development of novel

405 antimycotics. Most fungal pathogens of mammals are saprophytes that generally thrive in soil or

406 decaying organic matter, but can opportunistically cause disease in immunocompromised patients

407 (52-54). Azoles are an important class of antifungal agents that are used to treat fungal infections in

408 humans. Unfortunately, agricultural practices involving massive spraying of azoles to control fungal

409 plant pathogens, but also the extensive use of azoles in personal care products, ultraviolet

410 stabilizers, and anti-corrosives in aircrafts, for instance, give rise to an enhanced evolution of azole

411 resistance in opportunistic pathogens of mammals in the environment $(52,55)$. For instance, azole

412 resistant Aspergillus fumigatus strains are ubiquitous in agricultural soils and in decomposing crop

413 waste material where they thrive as saprophytes $(56,57)$. Thus, fungal pathogens of mammals, like

414 A. fumigatus, comprise niche competitors of fungal plant pathogens. Hence, we speculate that, like

$415 \quad V$. dahliae, also other plant pathogenic fungi may carry potent antifungal proteins in their effector

416 catalogues that aid in niche competition with these fungi. Possibly, the identification of such

417 effectors could contribute to the development of novel antimycotics. 


\section{MATERIALS AND METHODS}

\section{Gene expression analyses}

420 In vitro cultivation of $V$. dahliae strain JR2 for analysis of VdAMP3 and Chr6g02430 expression was

421 performed as described previously (24). Additionally, for in planta expression analyses, total RNA

422 was isolated from individual leaves or complete $N$. benthamiana plants harvested at different time

423 points after $V$. dahliae root dip inoculation. To induce microsclerotia formation, $N$. benthamiana

424 plants were harvested at $22 \mathrm{dpi}$ and incubated in sealed plastic bags (volume $=500 \mathrm{~mL}$ ) for 8 days,

425 prior to RNA isolation. RNA isolations were performed using the Maxwell ${ }^{8} 16$ LEV Plant RNA Kit

426 (Promega, Madison, USA). Real-time PCR was performed as described previously using the primers

427 listed in Supplementary Table 3 (17).

428

Generation of $V$. dahliae mutants

430 The VdAMP3 deletion and complementation mutants, as well as the eGFP expression mutant, were 431 generated as described previously using the primers listed in Supplementary Table 3 (18). To 432 generate the VdAMP3 complementation construct, the VdAMP3 coding sequence was amplified 433 with flanking sequences ( $\sim .9 \mathrm{~kb}$ upstream and $\sim 0.8 \mathrm{~kb}$ downstream) and cloned into pCG (58). 434 Finally, the construct was used for Agrobacterium tumefaciens-mediated transformation of $V$. 435 dahliae as described previously (59). In vitro growth and microsclerotia production of the VdAMP3 436 deletion mutant was tested and quantified as described previously (18).

439 Bacterial strains B. subtilis AC95, S. xylosus M3, P. corrugata C26, Streptomyces sp. NE-P-8 and 440 Ralstonia sp. M21 were obtained from our in-house endophyte culture collection. Bacterial strains 441 Novosphingobium sp. (NCCB 100261) and Sphingobacterium canadese (NCCB100125) were obtained 442 from the Westerdijk Fungal Biodiversity Institute (Utrecht, the Netherlands). Fungal strains 443 Saccharomyces cerevisiae $\mathrm{H} 15$ and Trichoderma viride were obtained from our in-house culture 
444 collection. Fungal strains Cyberlindnera jadinii (DSM 70167), Cordyceps militaris (DSM 1153),

445 Debaryomyces vanrijiae (DSM 70252), Meyerozyma amylolytica (DSM 27310) and Rhodotorula

446 bogoriensis (DSM 70872) were obtained from the Leibniz Institute DSMZ.

In vitro microbial growth assays

449 Bacterial isolates were grown on lysogeny broth agar (LBA) at $28^{\circ} \mathrm{C}$. Single colonies were selected 450 and grown overnight in low salt LB (10 g/L tryptone, $5 \mathrm{~g} / \mathrm{L}$ yeast extract and $0.5 \mathrm{~g} / \mathrm{L}$ sodium chloride) 451 at $28^{\circ} \mathrm{C}$ while shaking at $200 \mathrm{rpm}$. Overnight cultures were resuspended to $\mathrm{OD}_{600}=0.025$ in fresh low 452 salt LB supplemented with $20 \mu \mathrm{M}$ VdAMP3 or ultrapure water (MQ). In vitro growth was quantified using a CLARIOstar plate reader (BMG Labtech) as described previously (18). colonies were selected and grown overnight in $0.05 x$ potato dextrose broth (PDB) at $28^{\circ} \mathrm{C}$ while shaking at $200 \mathrm{rpm}$. Overnight cultures were resuspended to $O D_{600}=0.01$ in fresh $0.05 \mathrm{x}$ potato dextrose broth supplemented with $5 \mu \mathrm{M}$ VdAMP3 or ultrapure water (MQ). Alternatively, for filamentous fungi, spores were harvested from PDA and suspended in $0.05 x$ potato dextrose broth supplemented with $5 \mu \mathrm{M}$ VdAMP3 or ultrapure water (MQ) to a final concentration of $10^{4}$ spores $/ \mathrm{mL}$. Next, $200 \mu \mathrm{L}$ of the fungal suspensions was aliquoted in clear 96-well flat-bottom

461 polystyrene tissue culture plates. Plates were incubated at $28^{\circ} \mathrm{C}$ and fungal growth was imaged using 462 a SZX10 stereo microscope (Olympus) with EP50 camera (Olympus).

465 Three-week-old $N$. benthamiana seedlings grown in the greenhouse at $21^{\circ} \mathrm{C} / 19^{\circ} \mathrm{C}$ during $16 \mathrm{~h} / 8 \mathrm{~h}$ 466 day/night periods, respectively, with $70 \%$ relative humidity, were inoculated with $V$. dahliae through 467 root-dip inoculation as described previously (60). After 14 days, above-ground parts of the $N$. 468 benthamiana plants were harvested and stored at $-20^{\circ} \mathrm{C}$. Alternatively, above-ground parts were collected and transferred to plastic bags (volume $=500 \mathrm{~mL}$ ) and incubated for four weeks at room 
470 temperature. Next, all $N$. benthamiana samples were ground using mortar and pestle. Subsequent

471 genomic DNA isolation and $V$. dahliae biomass quantification was performed as previously described 472 using the primers listed in Supplementary Table 3 (61).

\section{Fluorescence microscopy}

475 Conidiospores of the pVIAMP3::eGFP reporter strain were harvested from a PDA plate and diluted 476 to a final concentration of $10^{5}$ conidiospores $/ \mathrm{mL}$ in $0.1 \times$ Czapek Dox medium. The suspension was 477 incubated for one week at room temperature to allow hyphae to grow and microsclerotia to form. 478 Finally, eGFP accumulating in the fungal cells was detected using a Nikon ECLIPSE 90i microscope.

\section{Microbiome analysis}

481 Inoculation and incubation of $N$. benthamiana plants was performed as described above. After four 482 weeks of incubation in plastic bags at room temperature in the dark, the decaying $N$. benthamiana 483 phyllosphere samples colonized by V. dahliae WT and the VdAMP3 deletion mutant were collected.

484 The phyllospheres of fresh three-week-old $N$. benthamiana plants were included as controls. All 485 samples were flash-frozen in liquid nitrogen and ground using mortar and pestle, genomic DNA was 486 isolated using the DNeasy PowerSoil Kit (Qiagen, Venlo, The Netherlands). Sequencing libraries were 487 prepared using the TruSeq DNA Nano kit (Illumina, San Diego, CA) and paired-end 150 bp sequencing 488 was performed on the Illumina NextSeq500 platform at the Utrecht Sequencing Facility (USEQ).

489 The sequencing data was processed as follows. Quality control of the reads, adapter 490 trimming and removal of $N$. benthamiana reads was performed with the ATLAS metagenomic 491 workflow using the default parameters of the configuration file (62). Reads of the different samples 492 were combined and assembled using metaSPAdes (used k-mer sizes: 21, 33, 55) to obtain a single metagenome cross-assembly (63). Subsequently, the cross-assembled contigs were taxonomically classified using CAT and binned per genus (64). The reads of the individual samples were mapped to 
496 using SAMtools (66) v1.10 and the number of reads mapped to the contigs of a single genus were

497 converted to "reads per million" for the individual samples. The generated taxonomy table and

498 abundance table were subsequently transformed into a phyloseq (67) object (v.1.30.0) in R (v.3.6.1)

499 to facilitate analysis of the microbiomes. The alpha diversity (Shannon index) and beta diversity

500 (Bray-Curtis dissimilarity) were determined as described previously $(67,68)$. The DESeq2 extension

501 of phyloseq was used to identify differentially abundant microbial genera (69). To this end, a

502 parametric model was applied to the data and a negative binomial Wald test was used to test for

503 significant differential abundance.

504

\section{Fungal co-cultivation assays}

506 Fungal isolates were grown on PDA at room temperature. For D. vanrijiae and M. amylolytica single

507 colonies were selected and grown overnight in $0.05 x$ PDB at $28^{\circ} \mathrm{C}$ while shaking at $200 \mathrm{rpm}$. The

508 overnight cultures of $D$. vanrijiae and $M$. amylolytica were resuspended to $\mathrm{OD}_{600}=0.001$ and 0.0001

509 in fresh $0.05 \times$ PDB, respectively. Conidiospores of $V$. dahliae strain JR2 and the VdAMP3 deletion

510 mutant were harvested from PDA plates and diluted in ultrapure water (MQ) to a final concentration

511 of $10^{4}$ conidiospores $/ \mathrm{mL}$. Next, $150 \mu \mathrm{L}$ of the yeast suspensions were mixed with $150 \mu \mathrm{L}$ of the $V$.

512 dahliae condiospore suspensions in clear 24-well flat-bottom polystyrene tissue culture plates.

513 Finally, after seven days of incubation at $22^{\circ} \mathrm{C}$, fungal growth was imaged using a SZX10 stereo

514 microscope (Olympus) with EP50 camera (Olympus). The number of microsclerotia formed by $V$.

515 dahliae WT and the VdAMP3 deletion mutant was quantified using ImageJ. 


\section{Acknowledgements}

517 B.P.H.J.T. is supported by the Research Council Earth and Life Sciences (ALW) of the Netherlands

518 Organization of Scientific Research (NWO). B.P.H.J.T acknowledges funding by the Alexander von

519 Humboldt Foundation in the framework of an Alexander von Humboldt Professorship endowed by

520 the German Federal Ministry of Education and research is furthermore supported by the Deutsche

521 Forschungsgemeinschaft (DFG, German Research Foundation) under Germany's Excellence Strategy

522 - EXC 2048/1 - Project ID: 390686111. We thank Utrecht Sequencing Facility, subsidized by the

523 University Medical Center Utrecht, Hubrecht Institute, Utrecht University, and The Netherlands X-

524 omics Initiative (NWO project 184.034.019), for providing sequencing service. The authors declare

525 no competing interests exist.

\section{Author contributions}

528 N.C.S. and B.P.H.J.T. conceived the project. N.C.S., G.C.P. and B.P.H.J.T. designed the experiments.

529 N.C.S., G.C.P. and G.C.M.B. carried out the experiments. N.C.S., G.C.P., M.F.S. and B.P.H.J.T. analyzed

530 the data. N.C.S. and B.P.H.J.T. wrote the manuscript. All authors read and approved the final

531 manuscript.

532

533 Data and mate rials availability

534 The metagenomics data have been deposited in the NCBI GenBank database under BioProject

535 PRJNA728211.

536 


\section{REFERENCES}

538 1. F. Demoling, D. Figueroa, E. Bååth, Comparison of factors limiting bacterial growth in 539 different soils. Soil Biol. Biochem. 39, 2485-2495 (2007).

540 2. L. Katz, R. H. Baltz, Natural product discovery: past, present, and future. J. Ind. Microbiol. $541 \quad$ Biotechnol. 43, 155-176 (2016).

542 3. A. Van der Meij, S. F. Worsley, M. I. Hutchings, G. P. van Wezel, Chemical ecology of antibiotic production by actinomycetes. FEMS Microbiol. Rev. 41, 392-416 (2017).

4. H. Rovenich, J. C. Boshoven, B. P. H. J. Thomma, Filamentous pathogen effector functions: of pathogens, hosts and microbiomes. Curr. Opin. Plant Biol. 20, 96-103 (2014).

5. N. C. Snelders, G. J. Kettles, J. J. Rudd, B. P. H. J. Thomma, Plant pathogen effector proteins as manipulators of host microbiomes? Mol. Plant Pathol. 19, 257 (2018).

6. M. C. Giraldo, B. Valent, Filamentous plant pathogen effectors in action. Nat. Rev. Microbiol. 11, 800-814 (2013).

7. L. Lo Presti, et al., Fungal effectors and plant susceptibility. Annu. Rev. Plant Biol. 66, 513-545 (2015).

8. R. L. Berendsen, C. M. J. Pieterse, P. A. H. M. Bakker, The rhizosphere microbiome and plant health. Trends Plant Sci. 17, 478-486 (2012).

9. C. Dimkpa, T. Weinand, F. Asch, Plant-rhizobacteria interactions alleviate abiotic stress conditions. Plant. Cell Environ. 32, 1682-1694 (2009).

10. G. Castrillo, et al., Root microbiota drive direct integration of phosphate stress and immunity. Nature 543, 513-518 (2017).

11. C. R. Fitzpatrick, et al., Assembly and ecological function of the root microbiome across angiosperm plant species. Proc. Natl. Acad. Sci. 115, E1157-E1165 (2018).

12. Y. Bai, et al., Functional overlap of the Arabidopsis leaf and root microbiota. Nature 528, 364369 (2015).

13. P. Durán, et al., Microbial interkingdom interactions in roots promote Arabidopsis survival. Cell 175, 973-983 (2018).

14. N. Lombardi, et al., Root exudates of stressed plants stimulate and attract Trichoderma soil fungi. Mol. Plant-Microbe Interact. 31, 982-994 (2018).

15. R. L. Berendsen, et al., Disease-induced assemblage of a plant-beneficial bacterial consortium. ISME J. 12, 1496-1507 (2018).

16. V. J. Carrión, et al., Pathogen-induced activation of disease-suppressive functions in the endophytic root microbiome. Science. 366, 606-612 (2019).

17. R. de Jonge, et al., Tomato immune receptor Ve1 recognizes effector of multiple fungal pathogens uncovered by genome and RNA sequencing. Proc. Natl. Acad. Sci. 109, 5110-5115 (2012).

18. N. C. Snelders, et al., Microbiome manipulation by a soil-borne fungal plant pathogen using effector proteins. Nat. Plants 6, 1365-1374 (2020).

19. P. Inderbitzin, et al., Phylogenetics and taxonomy of the fungal vascular wilt pathogen Verticillium, with the descriptions of five new species. PLoS One 6 (2011).

20. S. J. Klosterman, et al., Comparative genomics yields insights into niche adaptation of plant vascular wilt pathogens. PLoS Pathog. 7 (2011).

21. L. Mol, H. W. Van Riessen, Effect of plant roots on the germination of microsclerotia of Verticillum dahliae. Eur. J. plant Pathol. 101, 673-678 (1995).

22. S. J. Klosterman, Z. K. Atallah, G. E. Vallad, K. V Subbarao, Diversity, pathogenicity, and management of Verticillium species. Annu. Rev. Phytopathol. 47, 39-62 (2009).

23. W. C. Schnathorst, Life cycle and epidemiology of Verticillium. Fungal wilt Dis. plants 82 (1981).

24. D. Xiong, et al., Deep mRNA sequencing reveals stage-specific transcriptome alterations during microsclerotia development in the smoke tree vascular wilt pathogen, Verticillium dahliae. BMC Genomics 15, 324 (2014). 
588

589

590

591

592

593

594

595

596

597

598

599

600

601

602

603

604

605

606

607

608

609

610

611

612

613

614

615

616

617

618

619

620

621

622

623

624

625

626

627

628

629

630

631

632

633

634

635

636

637

638
25. R. de Oliveira Dias, O. L. Franco, Cysteine-stabilized $\alpha \beta$ defensins: from a common fold to antibacterial activity. Peptides 72, 64-72 (2015).

26. L. A. Kelley, S. Mezulis, C. M. Yates, M. N. Wass, M. J. E. Sternberg, The Phyre2 web portal for protein modeling, prediction and analysis. Nat. Protoc. 10, 845 (2015).

27. T. M. A. Shafee, F. T. Lay, M. D. Hulett, M. A. Anderson, The defensins consist of two independent, convergent protein superfamilies. Mol. Biol. Evol. 33, 2345-2356 (2016).

28. M. Dal Peraro, F. G. Van Der Goot, Pore-forming toxins: ancient, but never really out of fashion. Nat. Rev. Microbiol. 14, 77-92 (2016).

29. P. H. Mygind, et al., Plectasin is a peptide antibiotic with therapeutic potential from a saprophytic fungus. Nature 437, 975-980 (2005).

30. T. Schneider, et al., Plectasin, a fungal defensin, targets the bacterial cell wall precursor Lipid II. Science. 328, 1168-1172 (2010).

31. J. S. Oeemig, et al., Eurocin, a new fungal defensin: structure, lipid binding, and its mode of action. J. Biol. Chem. 287, 42361-42372 (2012).

32. I. V Grigoriev, et al., MycoCosm portal: gearing up for 1000 fungal genomes. Nucleic Acids Res. 42, D699-D704 (2014).

33. J. W. Spatafora, et al., A phylum-level phylogenetic classification of zygomycete fungi based on genome-scale data. Mycologia 108, 1028-1046 (2016).

34. S. Kumar, G. Stecher, M. Suleski, S. B. Hedges, TimeTree: a resource for timelines, timetrees, and divergence times. Mol. Biol. Evol. 34, 1812-1819 (2017).

35. D. S. Heckman, et al., Molecular evidence for the early colonization of land by fungi and plants. Science. 293, 1129-1133 (2001).

36. M. L. Berbee, J. W. Taylor, "Fungal molecular evolution: gene trees and geologic time" in Systematics and Evolution, (Springer, 2001), pp. 229-245.

37. J. L. Morris, et al., The timescale of early land plant evolution. Proc. Natl. Acad. Sci. 115, E2274-E2283 (2018).

38. L. Faino, R. de Jonge, B. P. H. J. Thomma, The transcriptome of Verticillium dahliae infected Nicotiana benthamiana determined by deep RNA sequencing. Plant Signal. Behav. 7, 10651069 (2012).

39. J. Depotter, et al., Homogenization of sub-genome secretome gene expression patterns in the allodiploid fungus Verticillium longisporum. BioRxiv, 341636 (2018).

40. H. Gibriel, J. Li, L. Zhu, M. F. Seidl, B. P. H. J. Thomma, Verticillium dahliae strains that infect the same host plant display highly divergent effector catalogs. bioRxiv, 528729 (2019).

41. D. Duressa, et al., RNA-seq analyses of gene expression in the microsclerotia of Verticillium dahliae. BMC Genomics 14, 607 (2013).

42. A. Klimes, K. F. Dobinson, A hydrophobin gene, VDH1, is involved in microsclerotial development and spore viability in the plant pathogen Verticillium dahliae. Fungal Genet. Biol. 43, 283-294 (2006).

43. H. C. Smith, The morphology of Verticillium albo-atrum, V. dahliae, and V. tricorpus. New Zeal. J. Agric. Res. 8, 450-478 (1965).

44. M. R. Mäkelä, N. Donofrio, R. P. de Vries, Plant biomass degradation by fungi. Fungal Genet. Biol. 72, 2-9 (2014).

45. J. Vořišková, P. Baldrian, Fungal community on decomposing leaf litter undergoes rapid successional changes. ISME J. 7, 477-486 (2013).

46. T. Korkama-Rajala, M. M. Müller, T. Pennanen, Decomposition and fungi of needle litter from slow-and fast-growing Norway spruce (Picea abies) clones. Microb. Ecol. 56, 76 (2008).

47. T. Osono, Role of phyllosphere fungi of forest trees in the development of decomposer fungal communities and decomposition processes of leaf litter. Can. J. Microbiol. 52, 701-716 (2006).

48. T. Osono, Phyllosphere fungi on leaf litter of Fagus crenata: occurrence, colonization, and succession. Can. J. Bot. 80, 460-469 (2002). 
49. R. Sapkota, K. Knorr, L. N. Jørgensen, K. A. O'Hanlon, M. Nicolaisen, Host genotype is an important determinant of the cereal phyllosphere mycobiome. New Phytol. 207, 1134-1144 (2015).

50. T. Gomes, J. A. Pereira, J. Benhadi, T. Lino-Neto, P. Baptista, Endophytic and epiphytic phyllosphere fungal communities are shaped by different environmental factors in a Mediterranean ecosystem. Microb. Ecol. 76, 668-679 (2018).

51. A. E. Arnold, Understanding the diversity of foliar endophytic fungi: progress, challenges, and frontiers. Fungal Biol. Rev. 21, 51-66 (2007).

52. P. E. Verweij, E. Snelders, G. H. J. Kema, E. Mellado, W. J. G. Melchers, Azole resistance in Aspergillus fumigatus: a side-effect of environmental fungicide use? Lancet Infect. Dis. 9, 789-795 (2009).

53. R. C. May, N. R. H. Stone, D. L. Wiesner, T. Bicanic, K. Nielsen, Cryptococcus: from environmental saprophyte to global pathogen. Nat. Rev. Microbiol. 14, 106-117 (2016).

54. E. Klein, M. Ofek, J. Katan, D. Minz, A. Gamliel, Soil suppressiveness to Fusarium disease: shifts in root microbiome associated with reduction of pathogen root colonization. Phytopathology 103, 23-33 (2013).

55. J. B. Buil, et al., The fading boundaries between patient and environmental routes of triazole resistance selection in Aspergillus fumigatus. PLoS Pathog. 15, e1007858 (2019).

56. J. Zhang, et al., Dynamics of Aspergillus fumigatus in azole fungicide-containing plant waste in the Netherlands (2016-2017). Appl. Environ. Microbiol. 87 (2021).

57. S. E. Schoustra, et al., Environmental hotspots for azole resistance selection of Aspergillus fumigatus, the Netherlands. Emerg. Infect. Dis. 25, 1347 (2019).

58. L. Zhou, J. Zhao, W. Guo, T. Zhang, Functional analysis of autophagy genes via Agrobacteriummediated transformation in the vascular wilt fungus Verticillium dahliae. J. Genet. genomics 40, 421-431 (2013).

59. P. Santhanam, "Random insertional mutagenesis in fungal genomes to identify virulence factors" in Plant Fungal Pathogens, B. P. H. J. Thomma, M. D. Bolton, Eds. (Springer, 2012), pp. 509-517.

60. E. F. Fradin, et al., Genetic dissection of Verticillium wilt resistance mediated by tomato Ve1. Plant Physiol. 150, 320-332 (2009).

61. Y. Song, et al., Transfer of tomato immune receptor Ve1 confers Ave1-dependent Verticillium resistance in tobacco and cotton. Plant Biotechnol. J. 16, 638-648 (2018).

62. S. Kieser, J. Brown, E. M. Zdobnov, M. Trajkovski, L. A. McCue, ATLAS: a Snakemake workflow for assembly, annotation, and genomic binning of metagenome sequence data. $B M C$ Bioinformatics 21, 1-8 (2020).

63. S. Nurk, D. Meleshko, A. Korobeynikov, P. A. Pevzner, metaSPAdes: a new versatile metagenomic assembler. Genome Res. 27, 824-834 (2017).

64. F. A. B. von Meijenfeldt, K. Arkhipova, D. D. Cambuy, F. H. Coutinho, B. E. Dutilh, Robust taxonomic classification of uncharted microbial sequences and bins with CAT and BAT. Genome Biol. 20, 1-14 (2019).

65. H. Li, Aligning sequence reads, clone sequences and assembly contigs with BWA-MEM. arXiv Prepr. arXiv1303.3997 (2013).

66. H. Li, et al., The sequence alignment/map format and SAMtools. Bioinformatics 25, 20782079 (2009).

67. P. J. McMurdie, S. Holmes, phyloseq: an R package for reproducible interactive analysis and graphics of microbiome census data. PLoS One 8, e61217 (2013).

68. B. J. Callahan, K. Sankaran, J. A. Fukuyama, P. J. McMurdie, S. P. Holmes, Bioconductor workflow for microbiome data analysis: from raw reads to community analyses. F1000Research 5 (2016).

69. M. I. Love, W. Huber, S. Anders, Moderated estimation of fold change and dispersion for RNA-seq data with DESeq2. Genome Biol. 15, 550 (2014). 


\section{$691 \quad$ FIGURE LEGENDS}

692 Figure 1. The $V$. dahliae effector VdAMP3 evolved from an ancient fungal protein. (a) VdAMP3

693 (left) is predicted to adopt a cysteine-stabilized $\alpha \beta(\operatorname{CS} \alpha \beta)$ defensin-like fold. The structure of the $694 \operatorname{CS} \alpha \beta$ defensin Plectasin (right) of the fungus Pseudoplectania nigrella is included as reference. The 695 disulfide bonds stabilizing the antiparallel $\beta$-sheets and the $\alpha$-helix are highlighted in yellow. 696 Positively and negatively charged amino acid residues are highlighted in blue and red, respectively. 697 (b) Protein sequence alignment with CS $\alpha \beta$ defensins Plectasin and Eurocin (Eurotium amstelodami) 698 supports the structure prediction of VdAMP3. (c) VdAMP3 homologs are widespread in the fungal 699 kingdom. Protein sequence alignment of VdAMP3 with a subset of its homologs identified in higher 700 (Ascomycota and Basidiomycota) and lower fungi (Mucoromycotina and Zoopagomycota). The

701 alignment as shown in (b-c) displays the most conserved region of the $\operatorname{CS} \alpha \beta$ defensin protein family 702 and was performed using HMMER and visualized with Espript3. The highly conserved cysteine and 703 glycine residues that contribute to the $\operatorname{CS} \alpha \beta$ defensin structure are highlighted by yellow and red 704 backgrounds, respectively. The homologs displayed in (c) were identified using blastP in the 705 predicted proteomes of the respective fungi included in the JGI 1000 Fungal Genomes Project (32).

Figure 2. VdAMP3 is specifically expressed in hyphal cells that develop into microsclerotia. (a)

708 Expression of VdAMP3 and the marker gene for microsclerotia development Chr6g02430, relative to 709 the household gene VdGAPDH at 48 and 96 hours of in vitro cultivation ( $\mathrm{N}=3$ ). (b) Expression of 710 VdAve1, VdAMP3 and Chr6g02430 in N. benthamiana leaves from 7 to 22 days post inoculation (dpi) $711(\mathrm{~N}=5)$. (c) Expression of VdAve1, VdAMP3 and Chr6g02430 in tissue of $N$. benthamiana plants 712 harvested at 22 days post inoculation after 8 days of incubation in sealed plastic bags ( $N=3)$. (d) 713 Microsclerotia formation of a pVdAMP3::eGFP reporter mutant as detected after 7 days of 714 cultivation in Czapek Dox medium. Typical chains of microsclerotia $(42,43)$ are indicated by arrows. 715 (e) Bright field image of various V. dahliae cell types after 7 days of cultivation in Czapek Dox, 716 including hyphae $(*)$, swollen hyphal cells developing into microsclerotia (‡) and mature 
717 microsclerotia cells (\#). (f) GFP signal for the image as shown in (e), indicative for activity of the

718 VdAMP3 promoter, is exclusively detected in the swollen hyphal cells developing into microsclerotia.

719 (g) Overlay of (e) and (f).

Figure 3. VdAMP3 is an antifungal protein that contributes to $V$. dahliae biomass accumulation in

the decaying host phyllosphere. (a) Microscopic pictures of fungal isolates grown in $0.05 x$ potato

dextrose broth supplemented with $5 \mu \mathrm{M}$ VdAMP3 or ultrapure water (MQ). VdAMP3 impairs growth

of Alternaria brassicicola, Cladosporium cucumerinum, Pichia pastoris and Saccharomyces cerevisiae.

Pictures were taken after 24 (A. brassicicola, C. cucumerinum and S. cerevisiae) or 64 (P. pastoris)

hours of incubation. (b) VdAMP3 does not contribute to establishment of Verticillium wilt disease in

$N$. benthamiana. Photos display representative phenotypes of $N$. benthamiana plants infected by

wild-type V. dahliae (WT), the VdAMP3 deletion ( $\triangle \mathrm{VdAMP3}$ ) and two complementation (Comp)

mutants 14 days post inoculation. (c) Relative $V$. dahliae biomass in above-ground $N$. benthamiana

tissues determined with real-time PCR. Different letter labels represent significant differences (one-

way ANOVA and Tukey's post-hoc test; $p<0.05 ; \mathrm{N} \geq 27$ (d) Representative phenotypes of $N$.

732 benthamiana plants as shown in (b) after 28 days of incubation in plastic bags. (e) Relative V. dahliae

733 biomass in N. benthamiana tissues as displayed in (d). Letters represent significant differences (one-

734 way ANOVA and Tukey's post-hoc test; $p<0.05 ; \mathrm{N} \geq 27$ ).

736 Figure 4. VdAMP3 manipulates the mycobiome of the decaying $\mathbf{N}$. benthamiana phyllosphere. (a-

737 b) $V$. dahliae-induced decay of the $N$. benthamiana phyllosphere is associated with a decreased

738 bacterial, and increased fungal, abundance. Relative abundance of bacteria (a) and fungi (b),

739 excluding $V$. dahliae, in the phyllosphere of decaying $N$. benthamiana plants colonized by wild-type

740 V. dahliae or the VdAMP3 deletion mutant (14 days post inoculation and after 28 days of incubation

741 in plastic bags), and in the phyllosphere of fresh $N$. benthamiana plants (mock). Letters represent

742 significant differences in total bacterial/fungal abundance between the three treatments (one-way 
743 ANOVA and Tukey's post hoc test; $P$ ? $<<0.05 ; \mathrm{N}=3$ ). (c) $V$. dahliae-induced decay of $N$. benthamiana

744 plants impacts alpha diversity of the phyllosphere. The plot displays the average Shannon index \pm

745 SD, letters represent significant differences (one-way ANOVA and Tukey's post hoc test; $P$ [?] 0.05 ;

$746 \mathrm{~N}=3$ ). (d) Principal coordinate analysis based on Bray-Curtis dissimilarities (beta diversity) reveals

747 separation of the microbiomes based on the three different treatments. (e) Differential abundance

748 analysis of microbial genera between the microbiomes colonized by $V$. dahliae WT and the VdAMP3

749 deletion mutant indicates that secretion of VdAMP3 significantly impacts a larger proportion of the

750 fungi than of the bacteria (two-tailed Fisher's exact test). (f) Of the differentially abundant microbial

751 genera, significantly more fungi display a decreased abundance in the presence of VdAMP3 when

752 compared with the bacteria (two-tailed Fisher's exact test). (g-h) Overview of the differentially

753 abundant bacterial (g) and fungal (h) genera. The plots display increased (positive $\log _{2}$ fold change)

754 or decreased (negative $\log _{2}$ fold change) abundance in the presence of $V$. dahliae WT when

755 compared with the VdAMP3 deletion mutant (Wald test, $P$ adjusted $<0.05$ and $P<0.05, N=3$ ).

756 Differentially abundant fungal genera from the Saccharomycetes or Sordariomycetes are

757 consistently suppressed in the presence of VdAMP3.

759 Figure 5. VdAMP3 contributes to $V$. dahliae microsclerotia formation in the presence of fungal

760 niche competitors. (a) Debaryomyces vanrijae and Meyerozyma amylolytica are inhibited by

761 VdAMP3. Microscopic pictures of the fungal species grown in $0.05 x$ potato dextrose broth

762 supplemented with $5 \mu \mathrm{M}$ VdAMP3 or ultrapure water (MQ). Pictures were taken after $10(D$.

763 vanrijae) or 24 (Meyerozyma amylolytica) hours of cultivation. (b) Close-up of V. dahliae

764 microsclerotia formed after seven days of cultivation in the presence of $D$. vanrijae or $M$.

765 amylolytica. (c) VdAMP3 contributes to V. dahliae microsclerotia formation in the presence of the

766 other fungal species. Representative microscopic pictures displaying the co-culture of $V$. dahliae with

767 D. vanrijae or M. amylolytica. Pictures were taken after seven days of co-cultivation. (d) Relative

768 number of microsclerotia formed by $V$. dahliae WT and the VdAMP3 deletion mutant in the 
bioRxiv preprint doi: https://doi.org/10.1101/2021.06.10.447847; this version posted June 10,2021. The copyright holder for this preprint (which was not certified by peer review) is the author/funder, who has granted bioRxiv a license to display the preprint in perpetuity. It is made available under aCC-BY 4.0 International license.

769 presence of D. vanrijae or M. amylolytica as determined using ImageJ (unpaired two-sided student's

$770 \quad$ t-test; $\mathrm{N}=3$ ). 